\title{
La carbonización como forma de encubrir un femicidio
}

\section{Carbonization process as a means to cover up a femicide}

\section{JV. Pachar Lucio}

M.Phil Forensic

Pathology.

Departamento de

Patología Forense

Instituto de Medicina

Legal y Ciencias.

Forenses de Panamá.

Ciudad de Panamá

Correspondencia:

José Vicente Pachar Lucio jvpachar@gmail.com

Fecha de recepción:

17. MAY. 2013

Fecha de aceptación

09. SEPT. 2013

\section{Resumen}

En la práctica de la patología forense es difícil y complejo establecer la identidad, la causa y la manera de la muerte en un cuerpo quemado hasta la carbonización. Los restos son frágiles y presentan múltiples artefactos, producto del trauma térmico, de factores ambientales y de su manipulación desde el lugar del hecho. Uno de los objetivos esenciales de la necropsia es determinar si el individuo estaba vivo o muerto al comenzar el incendio. Presentamos un caso de muerte por arma blanca y posterior quemadura del cuerpo hasta la carbonización, como intento de encubrir un femicidio.

Palabras clave: Reacción vital. Femicidio. Patología forense. Cuerpo carbonizado.

\section{Abstract}

In practical forensic pathology, it is difficult and complex to establish the identity, the cause and manner of death in a body burned up to carbonization. The remains are fragile and present multiple artifacts, product of the thermal trauma, of environmental factors and of its manipulation. One of the main objectives of the autopsy is to determine if the individual was alive or dead when the fire begun. We present a case of death by stab wound and posterior burn of the body up to carbonization as attempt of destroying the evidences of femicide.

Key words: Charred body. Vital reaction. Femicide. Forensic pathology.

\section{Presentación del caso}

El cadáver carbonizado de una mujer campesina de 68 años de edad, que vivía sola en un apartado lugar de la provincia de Panamá, fue encontrado entre los restos de una vivienda rural de madera, completamente quemada. El cuerpo fue remitido a la morgue judicial de ciudad de Panamá para su identificación y determinación de la causa de la muerte. No había registros de antecedentes patológicos de la fallecida.

\section{Examen necrópsico}

Se recibió un cuerpo incompleto, quemado hasta la carbonización, con amputaciones térmicas y pérdida de estructuras anatómicas en el tronco y los miembros (Figura 1). Fue identificado por sus características antropológicas y por la información recibida de los investigadores.

Al revisar las cavidades, las vísceras estaban bien conservadas. Había coágulos cocidos en el hemitórax izquierdo (Figura 2). En las vías aéreas no se encontraron partículas de carbón (Figura 3), y tampoco se observaron partículas de hollínal examen microscópico (Figura 4). El saco pericárdico estaba roto y contenía coágulos cocidos (Figura 5); al evacuarlos, se encontró una herida punzo-cortante, de $1,2 \times 0,8 \mathrm{~cm}$, que interesaba todo el espesor de la pared anterior del ventrículo izquierdo (Figuras 6 y 7). Al examen microscópico de la lesión se encontró fragmentación de fibras miocárdicas y hemorragia reciente (Figura 8 ). 


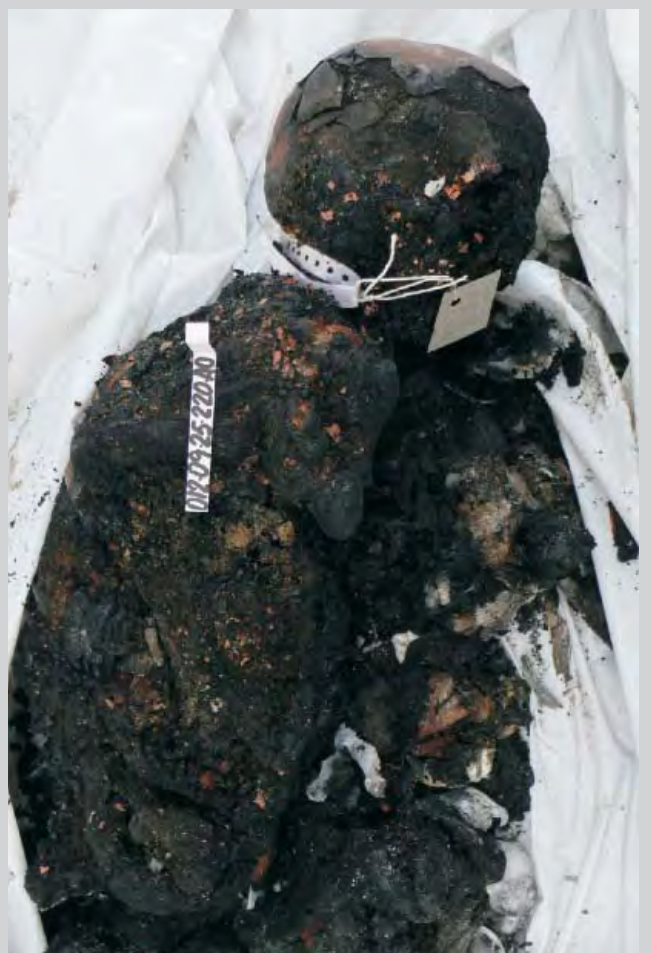

Figura 1.

Aspecto general del cuerpo.

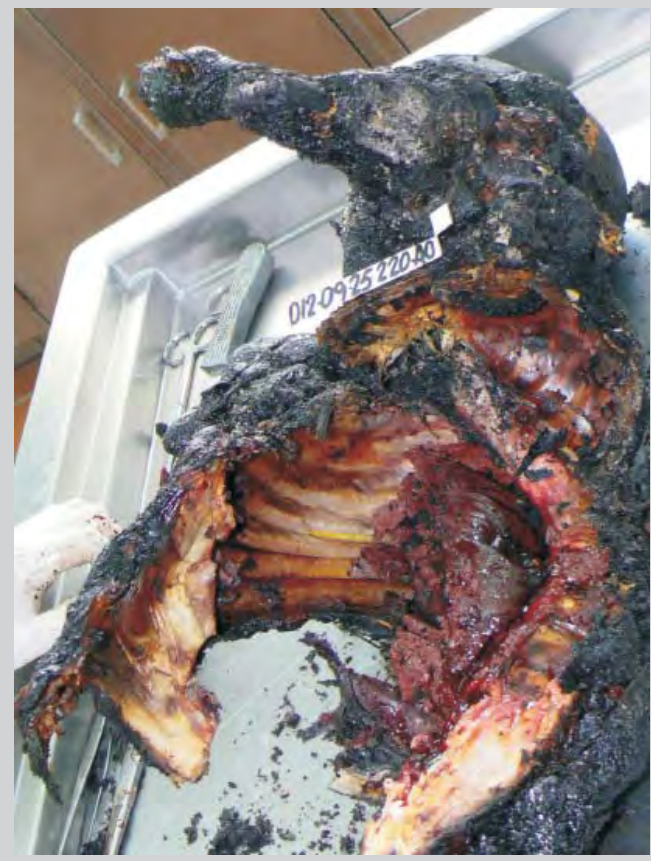

Figura 2.

Hemitórax izquierdo y contenido.

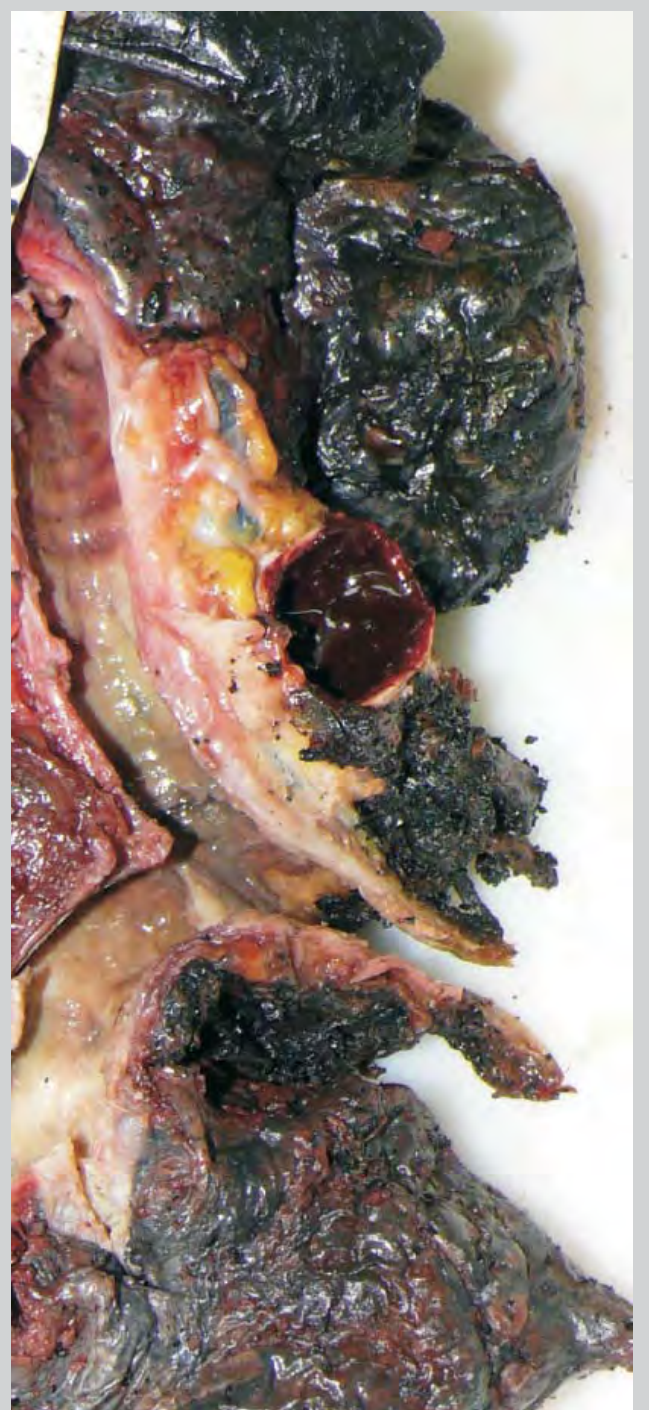

Figura 3.

Carina y bronquios principales.

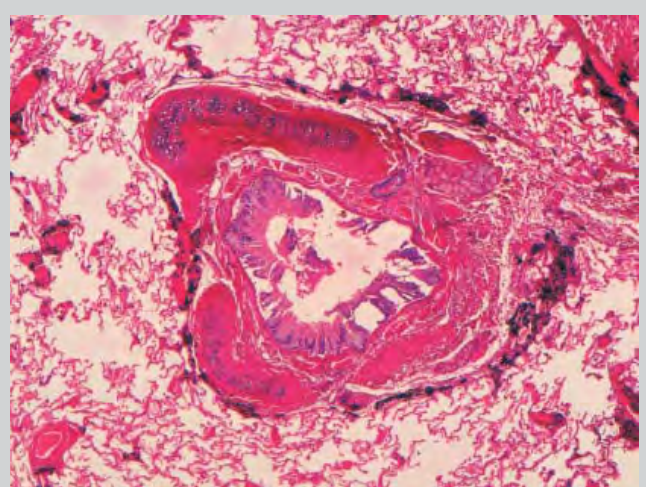

Figura 4.

Bronquiolo libre de hollín. 
Figura 5. Hemopericardio.

Figura 6. Herida punzo-cortante.
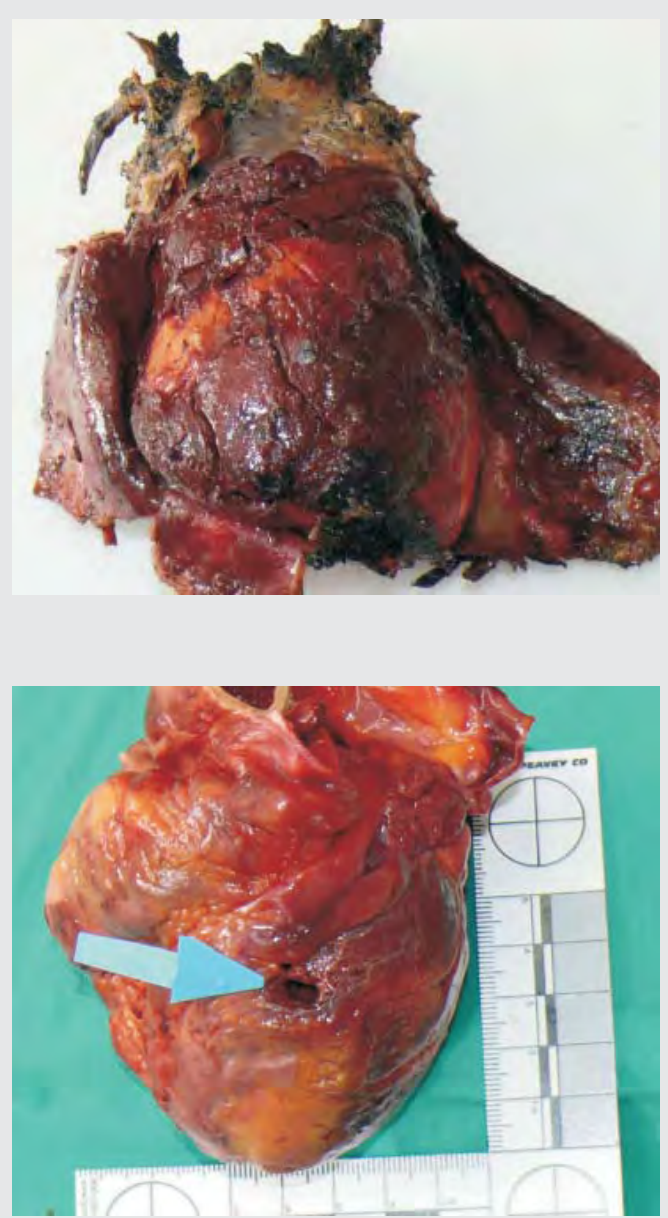

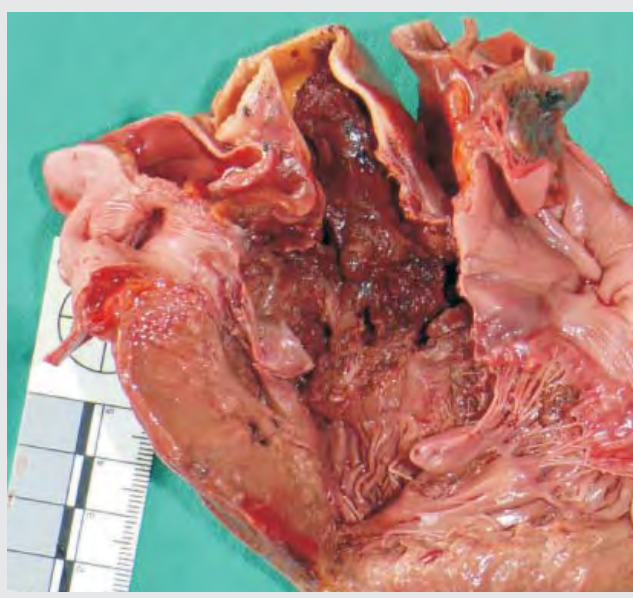

Figura 7.

Pared posterior del ventrículo izquierdo.

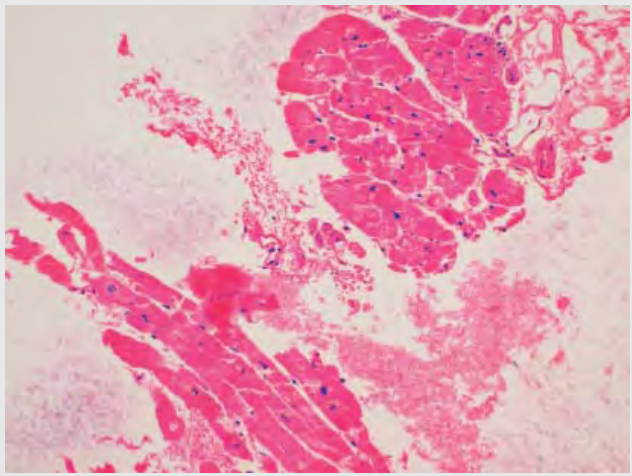

Figura 8.

Fibras miocárdicas fragmentadas. Hemorragia.
Se estableció que la causa de la muerte fue la herida punzo-cortante que interesó el corazón, y que el deceso ocurrió antes de la exposición térmica. Basándose en los resultados de la necropsia, los investigadores interrogaron a un familiar de la fallecida, quien confesó ser el autor del femicidio.

\section{Discusión}

En una vivienda incendiada, es inverosímil que el cuerpo de un adulto se queme por completo sin dejar rastros. La tentativa de encubrir una muerte intencional provocando el siniestro es infructuosa porque, a pesar de la importante destrucción corporal, y debido a su elevado contenido de agua, los órganos se conservan bastante bien y aún es posible obtener tejidos y fluidos corporales para análisis histopatológicos y de toxicología ${ }^{1-3}$.

El cuerpo carbonizado es frágil y presenta múltiples artefactos causados por el trauma térmico, los factores ambientales y su manipulación desde el lugar del hallazgo, razón por la cual en muchos de estos casos la causa de la muerte queda como indeterminada ${ }^{4,5}$.

El examen de estos cuerpos es un verdadero reto para el patólogo forense. Los objetivos principales de la necropsia son establecer la identidad y la causa y la manera de la muerte. Además, el médico deberá opinar sobre la posibilidad de que la persona estuviera muerta antes de iniciarse el incendio ${ }^{6-8}$.

El proceso de identificación incluye el análisis de toda la información ante-mórtem y de los hallazgos de la necropsia. En los cadáveres carbonizados, la certeza 
de la identidad se establece mediante los estudios de odontología y de genética forense (ADN) ${ }^{9}$.

Es difícil, sino imposible, distinguir macroscópicamente quemaduras ante o post mórtem, en especial en cuerpos carbonizados. Las pruebas de que la persona estaba viva en el momento de comenzar el incendio son la presencia de residuos negruzcos en las vías aéreas y una alta saturación sanguínea de monóxido de carbono; una prueba adicional es la documentación microscópica de una reacción vital en las quemaduras ${ }^{6,7}$.

El trauma térmico puede alterar o destruir lesiones preexistentes. El calor encoge los tejidos por la pérdida de agua y la coagulación de las proteínas, y debido a ello las heridas se reducen de tamaño y pueden pasar desapercibidas ${ }^{6,8}$; sin embargo, como en el presente caso, mediante una correcta realización de la necropsia pueden reconocerse y documentarse.

La quemadura intencional de un cuerpo, como modo de encubrir un homicidio, es la segunda, después de la forma accidental de trauma por exposición a altas temperaturas ${ }^{10}$. En el caso presentado, la víctima era una mujer (femicidio: asesinato de mujeres por razones asociadas al género ${ }^{11}$. El femicidio y el posterior intento de encubrimiento, siendo la estrangulación la causa de la muerte, ya se han descrito ${ }^{12}$.

En conclusión, pese a su aspecto, el examen minucioso de un cuerpo carbonizado aporta información de gran utilidad a la investigación.

Los autores declaran no tener conflicto de intereses.

\section{Bibliografía}

1. Spitz WU. Thermal injuries. Medicolegal investigation of death. 3rd ed. Springfield, Illinois, USA: Charles Thomas Publisher;1993. Cap. X.

2. Pachar JV. La necropsia en casos de incendio. Medicina legal y forense. Panamá: Artecsa; 2011.

3. Di Maio DJ, Di Maio VJM. Deaths due to fire. Forensic pathology. CRC Press. Boca Raton. USA Elsevier Publishing Co. Inc.;1993.

4. Tumer AR, et al. Postmortem burning of the corpses following homicide. Journal of Forensic and Legal Medicine. 2012;19:223-8.

5. Iwase $\mathrm{H}$, et al. Evidence for an antemortem injury of a burned head dissected froma burned body. Forensic Science International. 1998;94:9-14.

6. Schelover ER. Hallazgos anatomopatológicos en muertes por quemaduras en incendios. Cuadernos de los Ateneos Anatomo-Forenses. 2010;1:10-3.
7. Dolinak D, Matshes EW, Lew E. Enviromental injury. En: Forensic Pathology. Principles and Practice. San Diego, California, USA: Elsevier; 2005. p. 240-2.

8. Fanton L, et al. Criminal burning. Forensic Science International. 2006;158:87-93.

9. Cavard $S$, et al. Forensic and police identification of "X" bodies. A 6-years French experience. Forensic Science International. 2011;204:139-43.

10. Fanton L, et al. Criminal burning. Forensic Science International.2006;158:87-93.

11. Carcedo Cabanas A, Sagot Rodríguez M. Femicidio en Costa Rica: balance mortal. Med. Leg. Costa Rica [online]. 2002;19:5-16. (Consultado el 9/5/2013.) Disponible en: http://www.scielo. sa.cr/scielo.php?script =sci_arttext\&pid=S1409$00152002000100002 \& \operatorname{lng}=$ es\&nrm =iso > .

12. Suárez-Peñaranda JM, et al. Concealed homicidal strangulation by burning. Am JForensic Med and Pathology. 1999;20:141-4. 